\title{
Kernos
}

Revue internationale et pluridisciplinaire de religion grecque antique

19 | 2006

Varia

\section{Les catalogues de peuples sages}

Fonctions et contextes d'utilisation

Michèle Broze, Aude Busine et Sabrina Inowlocki

\section{Q OpenEdition \\ 1 Journals}

Édition électronique

URL : https://journals.openedition.org/kernos/437

DOI : $10.4000 /$ kernos.437

ISSN : 2034-7871

Éditeur

Centre international d'étude de la religion grecque antique

\section{Édition imprimée}

Date de publication : 1 janvier 2006

Pagination : 131-144

ISSN : 0776-3824

\section{Référence électronique}

Michèle Broze, Aude Busine et Sabrina Inowlocki, «Les catalogues de peuples sages », Kernos [En

ligne], 19 | 2006, mis en ligne le 20 mars 2011, consulté le 24 août 2022. URL : http://

journals.openedition.org/kernos/437; DOI : https://doi.org/10.4000/kernos.437 


\title{
Les catalogues de peuples sages Fonctions et contextes d'utilisation
}

\footnotetext{
Résumé : Cet article traite des catalogues des peuples perçus et représentés comme sages dans le monde grec, depuis leur genèse durant la période hellénistique jusqu'à leur exploitation par les chrétiens. Attentive à leurs dimensions chronologique, généalogique et topologique, cette étude se focalise sur les fonctions de ces catalogues. Elle met ainsi en exergue leur rôle dans les représentations de la transmission des savoirs ainsi que dans les débats interculturels et interreligieux des premiers siècles de notre ère.
}

\begin{abstract}
The Wise Peoples' Catalogues: Functions and Contexts of Use. This article deals with catalogues of peoples that are perceived and represented as wise in the Greek world, from their construction in the Hellenistic period until their exploitation by the Christians. By analysing their chronological, genealogical and topological dimensions, this paper focuses on the functions of these catalogues: it emphasises the role they played in the representations of the knowledge transmission process as well as in interreligious and intercultural debates in the Greco-Roman period.
\end{abstract}

La vénération de Platon pour les Barbares se montre à chaque instant; il se souvenait que lui-même, comme Pythagore, avait appris en pays barbare les plus nombreuses et les plus nobles de ses théories. Ainsi quand il dit 'les peuples barbares' veut-il dire 'peuples de philosophes barbares'.

Clément, Stromate I, 15, 68, 3

Si le catalogue s'exprime de manière linéaire, sans que l'ordre n'offre une logique immédiatement perceptible, sans construction dynamique ou narrative évidente pour le lecteur moderne, il n'en reste pas moins qu'aux yeux des anciens, il constitue une construction sémantique à part entière : rassemblant des termes initialement indépendants, le catalogue confère aux éléments qui le composent un sens nouveau, jouant un rôle actif dans la promotion d'une certaine vision du monde. Cette logique interne, qui ordonne et raisonne les catalogues, s'oppose de la sorte au caractère plus strictement exemplatif d'une simple liste.

Cette contribution à trois voix a pour objet les catalogues de peuples réputés pour leur sagesse, qui abondent dans la littérature grecque à partir de

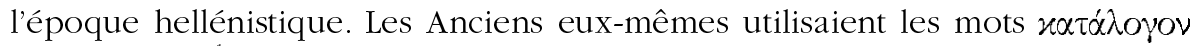
et $\varkappa \alpha \tau \alpha \lambda \alpha_{\gamma} \omega^{1} \omega^{1}$ pour évoquer ces listes de peuples souvent explicitement

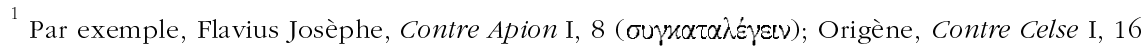

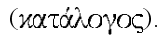




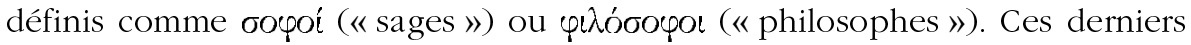
sont loués pour leur antiquité, leur sagesse, leur accès à la vérité, leurs connaissances, leurs pratiques rituelles, qu'elles soient magiques, astrologiques, théurgiques ou divinatoires, en somme lorsqu'il est question d'un contact privilégié avec le divin. Par convention, nous avons considéré comme catalogues de peuples sages les listes comprenant au minimum trois termes, et donc trois peuples.

L'examen de ces différents catalogues révèle la grande diversité des termes qui les composent. Certes, certains ensembles réapparaissent régulièrement, comme le noyau formé par les peuples orientaux (Égyptiens, Chaldéens et Assyriens sont fréquemment cités ensemble). Mais presque aucun catalogue n'est emprunté tel quel par un auteur à un autre. Cette absence d'homogénéité au sein des différents regroupements de peuples réputés pour leur sagesse rend l'étude des différents contextes d'exploitation capitale pour comprendre les diverses fonctions occupées par ces catalogues.

Les questions suscitées par les catalogues de peuples sages sont vastes, dans la mesure où leur exploitation par les Anciens a successivement été liée à la représentation des barbares dans le monde grec, à la transmission du savoir, puis au renversement du rapport de domination culturelle entre Grecs et Barbares opéré par les païens, les juifs et les chrétiens. Sans traiter de façon exhaustive ces diverses problématiques, cet article se propose d'exposer de quelle manière et à quelles fins les auteurs anciens, pour la plupart de langue grecque, ont eu recours à ces catalogues.

\section{La mise en catalogues des peuples sages}

\section{a) Genèse bellénistique}

Si, dès les débuts de la littérature grecque, Homère témoigne d'un intérêt pour les barbares et, si, à l'époque classique, les Grecs pensaient déjà avoir appris d'eux une grande partie de leurs idées religieuses et scientifiques ${ }^{2}$, ce n'est qu'à l'époque hellénistique que sont apparus les premiers catalogues de peuples sages à proprement parler. Les conquêtes d'Alexandre ont fait émerger un contexte politique et culturel propice à une nouvelle cartographie du monde habité. En effet, la propagation de la paideia dans des territoires non grecs a mené les héritiers de l'hellénisme à insister sur les qualités des peuples barbares nouvellement conquis dans des circonstances bien particulières.

Dès la fin du IV ${ }^{\mathrm{e}}$ siècle, Hécatée d'Abdère est chargé d'écrire une histoire de l'Égypte. Ce faisant, le conseiller de Ptolémée $\mathrm{I}^{\text {er }}$ applique de façon systématique la théorie faisant dépendre la culture et la religion grecques de l'Orient, en l'occurrence de l'Égypte. Le but de cette entreprise était éminem-

\footnotetext{
${ }^{2}$ Par exemple, Hérodote II, 81. Voir A.-J. Festugière, La révélation d'Hermès Trismégiste. I. L'astrologie et les sciences occultes, Paris, Lecoffre, 1949, p. 19-44; A. Momigliano, Alien Wisdom. The Limits of Hellenization, Cambridge, University Press, 1975; F. HARTOG, Le miroir d'Hérodote. Essai sur la représentation de l'autre, Paris, Gallimard, 1980.
} 
ment politique : d'une part, il s'agissait de fournir au pouvoir en place des outils efficaces pour contrôler les peuples dominés; de l'autre, la mise en valeur des Égyptiens permet d'attirer l'attention sur le royaume des Ptolémées dans un contexte d'âpre rivalité entre les différents royaumes des Diadoques.

Au III $^{\mathrm{e}}$ siècle avant notre ère, Mégasthène se voit de la même façon chargé d'écrire des Indika, d'une part pour fournir au lectorat grec des informations sur les mours d'un pays qui exerçait sur eux une réelle fascination, de l'autre pour servir les intérêts stratégiques, politiques et économiques du royaume séleucide ${ }^{3}$

Dans ce cadre, l'ambiance culturelle de l'époque, marquée par la mise en ordre du savoir grec traditionnel au sein de l'école aristotélicienne, joua certainement un rôle non négligeable dans la formation des premiers catalogues de peuples barbares réputés pour leur sagesse.

Une étape importante vers la constitution d'un catalogue de peuples sages réside dans le témoignage de Mégasthène ${ }^{4}$ : par le biais de la généalogie, Mégasthène fait des juifs et des philosophes indiens deux branches d'une même filiation. De la même manière, Cléarque de Soles, à la même époque, fait descendre les philosophes juifs des Indiens ${ }^{5}$.

Le plus ancien catalogue qui nous ait été transmis apparaît chez le péripatéticien Ératosthène, au III $^{\mathrm{e}}$ siècle avant notre ère ${ }^{6}$. Ce dernier s'oppose à la division bipartite du monde entre barbares et Grecs telle que l'élabora son maître Aristote, qui avait conseillé à Alexandre de traiter les barbares en ennemis et les Grecs en amis ${ }^{7}$. À la place, Ératosthène propose une division du

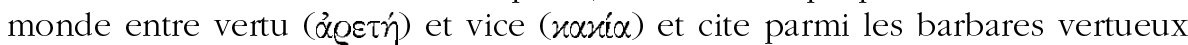
les Indiens, les Perses, les peuples de l'Ariane, les Romains et les Carthaginois.

Historiquement, ce procédé de mise en catalogue des peuples sages permettait d'assigner aux nouveaux barbares que l'on découvrait une place dans la représentation traditionnelle des contrées exotiques et de leurs sagesses.

\section{b) Critères d'inclusion}

Plusieurs critères régissaient l'inclusion de tel ou tel peuple au sein des catalogues de peuples sages.

\footnotetext{
${ }^{3}$ Voir G.E. STERLING, Historiography and Self-Definition, Josephos, Luke-Acts and Apologetic Historiography, Leyde, Brill, 1992 (Supplements to Novum Testamentum, 64), p. 59-78 et 92-102.

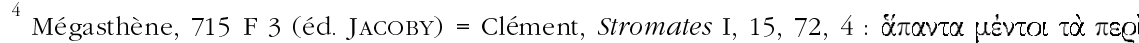

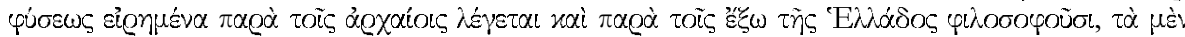

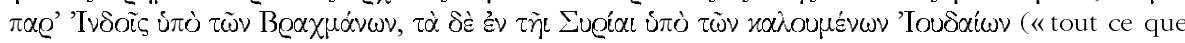
les anciens [grecs] ont dit sur la nature est dit aussi par les philosophes étrangers à la Grèce : soit aux Indes par les Brahmanes, soit en Syrie par ceux qu'on appelle les Juifs »).

5 Cléarque, fr. 5 (éd. Wehrli) = Jos., C. Ap. I, 179; F 13 (éd. WeHrL) = Diogène Laërce, Prologue 9

${ }^{6}$ Ératosthène, II C 24 (éd. JACOBY) = Strabon, I, 4, 9.

${ }^{7}$ Ps. Plutarque, De Alex. s. virt. s. fort., 1, 6 (Mor., 329b-c).
} 
Outre la traditionnelle fascination des Grecs pour l'Orient, il semble que la renommée d'un individu célèbre pour sa sagesse puisse avoir été, dans certains cas, à l'origine de l'intégration d'un peuple au catalogue de sagesse. Au même titre que Mochos chez les Phéniciens, Zalmoxis chez les Thraces ou Atlas chez les Libyens, il est probable que le Scythe Anacharsis, qui fut notamment compté parmi les Sept Sages de la Grèce?, soit la cause de la mention des Scythes parmi les peuples sages chez Clément et Augustin ${ }^{10}$.

Un autre critère d'inclusion réside dans des traits cultuels ou religieux, parfois fantasmés, mais qui justifient la promotion de certains peuples au rang de peuple sage. Par exemple, Numénius, dans son premier livre Sur le bien, évoque des peuples qui ont défini Dieu comme incorporel ${ }^{11}$. Ici, les peuples barbares ont été sélectionnés et promus au rang de sages en raison de la ressemblance présumée de leur définition du divin avec celle des monothéismes juif et chrétien.

La maîtrise de certaines sciences a aussi valu à différents peuples leur place dans ces catalogues. C'est notamment le cas de peuples orientaux : chez Strabon, Égyptiens, Chaldéens, Assyriens et Phéniciens sont réputés pour être à l'origine des sciences comme les mathématiques, l'astrologie, l'arithmétique ou la musique ${ }^{12}$. Cette surdétermination des sciences barbares est intimement liée au thème des "premiers inventeurs" ( les catalogues de peuples sages se voient associés à des catalogues d'inventeurs, qui mêlent individus et peuples ${ }^{14}$. Cela n'a rien de surprenant dans la mesure où l'on sait que la oọ̣íx désigne aussi la sagesse pratique.

Certains peuples ont encore été comptés comme sages en raison de leur mode de vie ascétique. C'est notamment le cas des Indiens (gymnosophistes, brahmanes ou sarmanes) et des juifs, et en particulier des Esséniens ${ }^{15}$.

Enfin, il faut souligner, au sein de ces catalogues, un phénomène de contamination, par métonymie, de la sagesse des castes sacerdotales de

\footnotetext{
${ }^{8}$ DL., Prol., 8. Voir aussi Clém., Str. I, 15, 1 qui rapporte des origines odryses à Orphée.

9 Voir J.F. KINDSTRAND, Anacharsis. The Legend and the Apophthegmata, Uppsala, 1981 (Studia Graeca Upsaliensia, 16); A. Busine, Les Sept Sages de la Grèce antique. Transmission et utilisation d'un patrimoine légendaire d'Hérodote à Plutarque, Paris, De Boccard, 2002, p. 25, 7273.

${ }^{10}$ Clém., Str. I, 15, 1; I, 14, 2; Augustin, De Ciuitate Dei VIII, 9.

${ }^{11}$ Numénius, fr. 1b (éd. DEs Places) = Orig., C. C. I, 15.

12 Str., XVI-XVII. Voir G. AUJAC, Strabon et la science de son temps, Paris, Les Belles Lettres, 1966, p. 105-112; G.E.R. LloYD, The Revolution of Wisdom. Studies in the Claims and Practice of Ancient Greek Science, Berkeley, California University Press, 1987, p. 51-56.

${ }^{13}$ Voir A. KLEINGÜNTHER, Protos heuretes. Untersuchungen zur Geschichte einer Fragestellung, Leipzig, Dieterich, 1933 (Philologus, Suppl. 26, 1); K. THRAEDE, s.v. « Erfinder», RAC 5 (1962), col. 1191-1278.

${ }_{15}^{14}$ Tatien, Oratio ad Graecos I, 1-3; Clém., Str. I, 16; Eusèbe, Préparation évangélique X, 1-2.

${ }^{15}$ Jos., Bellum Judaicum II, 119-159 = Porphyre, De abstinentia IV, 11. Pour les Indiens, voir J. ANDRÉ et A. Filliozat, L'Inde vue de Rome: textes latins de l'antiquité relatifs à l'Inde, Paris, 1986; J.-C. CARRIÈRE et al. (éd.), Inde, Grèce ancienne. Regards croisés en anthropologie de l'espace, Paris, Les Belles Lettres, 1995 (ALUB, 576. Centre de recherches d'bistoire ancienne, 148).
} 
certains peuples à l'ensemble de ces peuples eux-mêmes. En effet, des groupes sacerdotaux étrangers réputés pour leur sagesse ou pour leur pratique philosophique et/ou rituelle ont été rassemblés sous forme de catalogue. Ainsi, Cléarque de Soles explique que les philosophes sont appelés Calanoi chez les Indiens et juifs chez les Syriens ${ }^{16}$. Un Pseudo-Aristote et Sotion, tous deux traditionnellement situés au II $^{\mathrm{e}}$ siècle avant notre ère, incluent dans un catalogue de peuples de philosophes « les Mages des Perses, les Chaldéens des Babyloniens et des Assyriens, les Gymnosophistes des Indiens, et les Druides des Gaulois et des Celtes $\gg{ }^{17}$.

Les noms de ces classes sacerdotales se mêlent parfois aux seuls noms de peuples : au $\mathrm{I}^{\mathrm{er}}$ siècle avant notre ère, Cornutus présente côte à côte les mages d'une part, et de l'autre, « les Phrygiens, les Égyptiens, les Celtes, les Libyens et d'autres peuples ${ }^{18}$. Dans ce cas, la caste est devenue synonyme du nom de peuple, les mages désignant ici les Perses.

Découverts, vénérés, conquis, répertoriés, et enfin, catalogués, les peuples barbares pouvaient dès lors servir aux Anciens à situer leur civilisation dans l'espace et dans le temps.

\section{Catalogues et transmission du savoir}

La référence aux catalogues de peuples sages permettait d'expliquer symboliquement le cheminement de la sagesse grecque, ainsi que de retracer les modes de transmission du savoir. Cette exploitation des catalogues se faisait par la valorisation des dimensions tantôt chronologiques, tantôt généalogiques, tantôt topologiques de ces formes narratives.

Exploitant un schème généalogique bien particulier, celui de filiation intellectuelle, Eupolème, un auteur juif de la période hellénistique, fait remonter à Moïse et aux juifs la sagesse et l'écriture ${ }^{19}$. Ceux-ci les auraient ensuite transmises aux Phéniciens qui les auraient à leur tour transmises aux Grecs. Ce procédé vise souvent à glorifier le premier passeur d'un savoir, transmis d'un peuple à un autre. La position de dernier venu est attribuée ici aux Grecs, renforçant encore l'idée de l'origine barbare des sciences et de la sagesse au détriment de la culture grecque dominante.

Dans les écrits hermétiques, la sagesse est distribuée à certains peuples selon un ordre chronologique et en fonction d'espaces déterminés, par le biais de la triple incarnation du sage en différents endroits, avant ou après le

\footnotetext{
${ }^{16}$ Cléarq., fr. 5 (éd. Wehru) = Jos., C. Ap. I, 179; Cléarq., fr. 13 (éd. WeHrLi) = DL., Prol., 9.

${ }^{17}$ DL., Prol., 1 = Ps-Aristote, fr. 661 (éd. GIGON) = Sotion, fr. 35 (éd. WeHRLi).

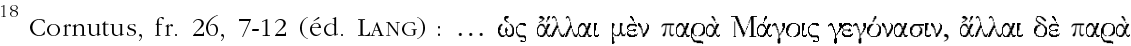

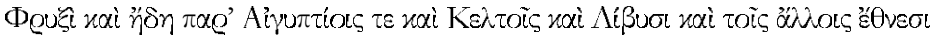

19 Alexandre Polyhistor, 672 F 3c (éd. JACOBY) = fr. 1 (éd. HolladAY) = Clém., Str. I, 153, $4=$ Eus., Prép. év. IX, 26.
} 
déluge $^{20}$. Cette organisation de la «descente » de la sagesse compense la perte du savoir, et en même temps l'universalise topologiquement et chronologiquement.

Par ailleurs, dans les textes relatifs à Pythagore et Apollonios de Tyane, c'est le schème du voyage qui construit la figure de l'homme sage ${ }^{21}$. Un être humain chemine littéralement dans le monde connu et visite les peuples sages dont il intègre la spécificité. En effet, l'apport des différents peuples catalogués dans ces voyages est différent. Dans ce modèle, la sagesse est éparpillée, morcelée dans le monde, et en parcourant par étapes le monde connu, le futur sage, fragment par fragment, réunifie ce qui était multiple et dispersé.

Une fois Pythagore établi en Italie, il devient le point central où affluent les autres peuples pour collecter la sagesse, ce qui leur donnera à eux aussi un statut de peuple sage dans d'autres listes. Il s'agit en quelque sorte d'une mise en scène du catalogue: en établissant avec le destinaire une proximité, il s'agit de «faire parler le monde », de le « rendre expressif »" Ces listes d'étapes traduisent l'effort exigé de l'homme pour retrouver l'unité d'un savoir, ce que le voyage exprime très bien, et le besoin de montrer la possibilité de ce savoir unifié en un seul être. Les pérégrinations de Pythagore fondent la possibilité pour l'homme de réunir ce qui était éparpillé, et dès lors incohérent. En cela, il est plus sage que les peuples sages, qui n'ont de la sagesse qu'une parcelle propre et ignorent les autres parcelles.

Le cas d'Apollonios est plus complexe, dans la mesure où ses voyages, qui lui permettent d'acquérir la sagesse, ne font géographiquement que le ramener à son point de départ le plus ultime, avant de le relancer dans les voyages. En parcourant les domaines des peuples sages, Apollonios, arrivé en Inde, en bon pythagoricien plus divin que le maître, se montre capable de se souvenir de sa première incarnation : celle d'un marin égyptien, dont le seul mérite fut de penser que la vertu, c'était s'abstenir de l'injustice. Le sage indien le raille pour cette conception, mais quand Apollonios aborde en Égypte, il rencontre à son tour un jeune marin égyptien, qui lui déclare qu'il ne suffit pas de s'abstenir d'injustice pour être sage. Apollonios, revenu sur les lieux de sa première incarnation, a lui aussi acquis dans son voyage chez les sages indiens cette étape supérieure de la sagesse. Aussi le voyage d'Apollonios chez les sages est-il double : c'est un voyage géographique, mais aussi

\footnotetext{
${ }^{20}$ Sur ce point voir M. BROZE, «Temps réel, temps imaginaire et temps fictionnel dans la révélation hermétique », in L. COuloubaritsis et J.J. Wunenburger (éd.), Figures du temps, Presses Universitaires de Strasbourg, 1997, p. 109-120.

${ }^{21}$ Voir les Vies de Pythagore de Porphyre, Jamblique et Diogène Laërce, et la Vie d'Apollonius de Tyane de Philostrate.

${ }^{22}$ Citations respectives de S. PERCEAU, La parole vive. Communiquer en catalogue dans l'épopée homérique, Leuven, 2002 et de L. Couloubaritsis, «Les enjeux du logos : de l'oral à l'écriture ", in M. BROzE et al. (éds), Oralité et écriture dans la pratique du mythe, Bruxelles, Presses Universitaires, 1998, p. 193-239.
} 
un voyage chronologique au fil de ses incarnations. Temps et espace s'associent pour construire le sage.

À l'inverse de ce qui se passe pour Pythagore ou Apollonios, qui cheminent pour unifier la sagesse, dans le De vita Mosis de Philon, ce sont les peuples orientaux, grecs et égyptiens, qui affluent de tout le pays et viennent à Moïse pour assurer la transmission. On note ici une hiérarchisation dans l'attitude des membres du catalogue et dans le contenu de leur savoir. En effet, les Grecs sont appelés à grands frais, mais les Égyptiens viennent spontanément, ce qui signifie qu'ils reconnaissent d'emblée la valeur de Moïse. D'autre part, les Égyptiens lui enseignent la philosophie symbolique qui s'exprime dans le culte, que Philon valorise chez les Thérapeutes. En bref, Moïse apprend des Égyptiens le sommet de la sagesse, mais les Égyptiens le lui livrent spontanément. Or, en ce qui concerne Pythagore ou Platon, la réticence des sages égyptiens est souvent mentionnée. On comprend dès lors que Moïse ne bouge pas, car vers lui déferle la sagesse des peuples, tandis que les sages grecs font eux-mêmes le voyage pour collecter la sagesse ${ }^{23}$.

Dans les cas étudiés précédemment, la sagesse morcelée s'unifie dans un individu. Dans les conceptions de l'empereur Julien, la répartition de la sagesse chez les différents peuples et son unification sont les fondements de sa pensée théologico-politique : à chaque peuple correspond une qualité propre présente parce que la Providence a réparti sur terre des dieux ethnarques $^{24}$. L'unité de ces qualités fait l'unité de l'Empire. Cette répartition topologique, liée à une permanence chronologique qui remonte au fond des temps, correspond bien au projet politico-religieux de Julien, pour qui « le polythéisme permet la reconnaissance de toutes les religions selon une topologie précise, qui correspond également au partage de l'Empire romain sous la tutelle de l'empereur ${ }^{25}$. De la même manière, la multiplicité des dieux est unifiée par un dieu un au sommet de la hiérarchie. Ce système exclut évidemment le judaïsme et le christianisme du catalogue.

Cette pensée politique qui fonde l'unité sur l'association providentielle des multiplicités trouve un parallèle chez Jamblique, sur le plan théologique et rituel, dans une défense de l'efficacité des rites païens.

En effet, dans le De Mysteriis, réponse à Porphyre aux critiques adressées à la théurgie, Jamblique, prenant dans la fiction la peau du prêtre égyptien Abamon, entend suivre l'inventaire des problèmes théologiques posés par Porphyre et y répondre de manière exhaustive à l'aide de trois sagesses, qui chacune correspondent à un type de problème : Assyriens (ou Chaldéens), Égyptiens et philosophes grecs. À chaque sagesse s'associe une méthode différente. Trois régions, trois peuples, trois méthodes pour aborder le rapport avec l'invisible. Cette énumération n'est pas statique. En effet, les philosophes

\footnotetext{
23 Philon, De Vita Mosis I, 23-24.

${ }^{24}$ Voir Julien, Contre les Galiléens, 115e - 116a et 176a-c (éd. GÉRARD).

${ }^{25}$ Voir L. Couloubaritsis, Histoire de la philosophie ancienne et médiévale, Paris, Grasset, 1998, p. $738-742$.
} 
grecs tirent clairement leur savoir du voyage en Égypte. Comme prêtre égyptien, le sage Abamon maîtrise donc les sagesses égyptienne et grecque, le seconde puisqu'il maittrise la première qui en est l'unique source valable. Pour y unir celle des Chaldéens, Jamblique-Abamon met en avant l'entretien privé qu'il eut avec un sage chaldéen. La fiction qu'il construit fait de lui le dépositaire de ces trois sagesses, et surtout des trois méthodes, qu'il hiérarchise et unifie. La philosophie grecque devient ainsi la propédeutique de la théologie, qui est celle de l'art théurgique.

Son catalogue des peuples sages, hiérarchisé, inclut la dimension chronologique et généalogique (filiation de maître à disciple) pour les Égyptiens et les Grecs, mais introduit de manière synchronique la sagesse chaldéenne, par le biais d'un entretien qui lui permet, à lui, d'inclure ce troisième pôle. Ainsi, Jamblique-Abamon pourrait lui aussi prétendre unifier en lui-même les qualités propres à chaque sagesse du catalogue $e^{26}$.

\section{Les catalogues au service de l'apologétique}

En contexte apologétique et polémique, la fonction essentielle des catalogues de peuples sages réside dans ce que les anglo-saxons désignent par selfdefinition: il s'agit de cartographier le «nous » par rapport au «ils » ou au «vous», et de poser la question cruciale du même et de l'autre ${ }^{27}$. Constituer un catalogue, c'est à la fois inclure les termes d'un ensemble et en exclure d'autres. Ces oppositions entre termes inclus et termes exclus du catalogue viennent nourrir une rhétorique de l'autre, qui varie étonnamment selon les auteurs et les contextes.

\section{a) Fonction unificatrice}

Les catalogues semblent d'abord avoir eu une fonction unificatrice. C'est la forme catalogique même qui permet le jeu d'équivalences entre les termes du catalogue. En effet, la juxtaposition des termes constitue une stratégie formelle efficace pour établir leur égalité. Dès le début, Ératosthène rejetait la dichotomie aristotélicienne entre Grecs et Barbares au profit d'une équivalence entre les peuples en fonction de critères éthiques ${ }^{28}$.

La fonction unificatrice des catalogues de peuples sages s'est particulièrement bien intégrée dans une démarche philosophique qui tendait, aux premiers siècles de notre ère, à unifier les traditions des Grecs et celles de certains peuples sages. C'est dans le stö̈cisme tardif et en particulier chez Cornutus qu'est née l'idée d'une sagesse primitive morcelée dans la mytholo-

\footnotetext{
${ }^{26}$ Voir M. BROZE, C. VAN LIEFFERINGE, «L'Hermès commun d'Abamon : philosophie grecque et théologie égyptienne dans le prologue du De mysteriis de Jamblique », Religions méditerranéennes et orientales de l'Antiquité, IFAO, Bibliothèque d'Etudes 135 (2002), p. 35-44.

${ }^{27}$ Voir STERLing, o.c. (n. 3); E.P. SANDERS, Jewish and Christian Self-Definition, Philadelphia, Fortress Press, 1980

${ }^{28}$ Eratosth., II C 24 (éd. JACOBY) = Str. I, 4, 9
} 
gie grecque et dans les mythologies des peuples sages ${ }^{29}$. Cette sagesse primitive est quasi-divine par son ancienneté, et donc sa proximité avec le divin. Le philosophe doit s'atteler à la retrouver, notamment par le biais de l'interprétation allégorique des mythes. Selon Cornutus, les mythes des Mages, des Phrygiens, des Égyptiens, des Celtes, des Libyens et d'autres races contiennent tous une parcelle de cette sagesse divine, qu'il s'agit de recouvrer par l'allégorèse ${ }^{30}$.

Héritiers de cette démarche, les platoniciens de l'époque romaine chercheront aussi à intégrer, notamment grâce au catalogue, les sagesses barbares dans leur pratique de la philosophie et leur quête de révélation divine. Un fragment de Numénius d'Apamée, philosophe du II è siècle de notre ère, illustre bien cette méthode : «après avoir cité et pris pour sceaux les témoignages de Platon, il faudra remonter plus haut et les rattacher aux enseignements de Pythagore, puis en appeler aux peuples de renom, en conférant leurs initiations, leurs dogmes, les fondations cultuelles qu'ils accomplissent en accord avec Platon, tout ce qu'ont établi les brahmanes, les juifs, les mages, et les Égyptiens » ${ }^{31}$ (trad. É. des Places).

Dans certains cas, la fonction unificatrice des catalogues de peuples sages, qui présentent côte à côte Grecs et Barbares, permet la promotion d'une sagesse et d'une communauté. Tel est le but de Philon dans le Probus ${ }^{32}$. En vantant la vertu et la civilité de peuples rangés en un catalogue qui pose sur le même pied les Sept sages, les mages perses, les gymnosophistes indiens et les Esséniens chez les juifs, Philon recherche manifestement moins à poser une équivalence de statut entre ces philosophes qu'à valoriser le peuple juif. Le catalogue peut donc servir en contexte apologétique à accorder à un peuple un statut équivalent à celui de la culture dominante. La manœuvre est d'autant plus discrète que le peuple en question est mêlé à d'autres peuples au sein du catalogue.

Certains chrétiens, sous l'influence du platonisme, ont à leur tour eu recours aux catalogues de peuples sages. Tout comme ces catalogues avaient permis à Philon de promouvoir le judaïsme au rang de philosophie, l'utilisation chrétienne de ces mêmes catalogues vise la légitimation philosophique du christianisme. Un extrait d'Augustin illustre particulièrement bien cette démarche :

Tous les philosophes quels qu'ils soient ont reconnu dans le Dieu suprême et véritable l'auteur des choses créées, la lumière de nos connaissances...; soit qu'on

\footnotetext{
${ }^{29}$ Voir G.R. BOYs-STONES, Post-Hellenistic Philosophy, A Study of Its Development from the Stoics to Origen, Oxford, University Press, 2001

${ }^{30}$ Cornutus, fr. 26, 7-12 (éd. LANG).

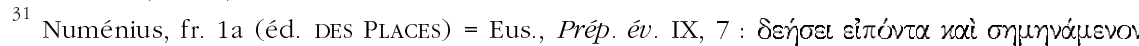

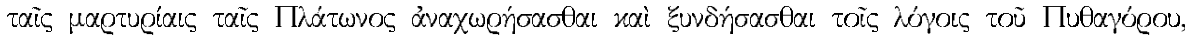

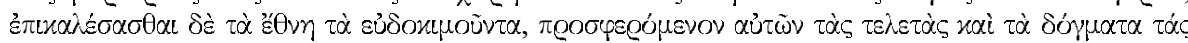

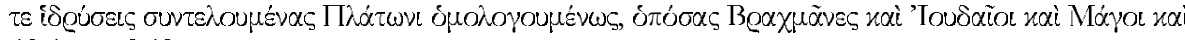

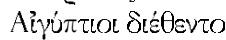

32 Phil., Quod omnis probus, 73-74.
} 
les appelle plus exactement platoniciens ou qu'on donne à leur école n'importe quel nom; soit qu'on estime que les maîtres les plus remarquables de l'école ionique, tels Platon..., ont été les seuls à penser ainsi; soit qu'on retrouve aussi cette doctrine dans l'école italique à cause de Pythagore...; soit qu'on découvre encore en d'autres nations des sages ou philosophes, Libyens atlantiques, Égyptiens, Indiens, Perses, Chaldéens, Scythes, Gaulois, Espagnols qui auraient saisi et enseigné ces doctrines, tous nous les plaçons au-dessus des autres et nous déclarons qu'ils sont plus près de nous ${ }^{33}$ (trad. G. Bardy).

En apparence, l'évêque d'Hippone ne cherche pas à rivaliser avec la philosophie grecque ou avec les peuples sages, mais il cherche plutôt à unifier ces traditions en tant qu'elles disposent de la même conception de Dieu que le christianisme. À y regarder de plus près, le message initié par Cornutus et récupéré par les néoplatoniciens est exploité ici par Augustin pour associer le christianisme au meilleur de la philosophie des peuples sages. Tel semble aussi être le cas d'un passage d'Arnobe qui inclut les Aegyptioi, Persai, Indoi, Chaldaeoi, Armenioi parmi les peuples sages adeptes du monothéisme ${ }^{34}$.

La fonction unificatrice du catalogue permet donc aux auteurs chrétiens d'effectuer une réappropriation des sagesses barbares vantées en milieu hellène. Il ne s'agit plus ici de recouvrer une sagesse primitive par le biais de diverses traditions, mais bien d'assurer le prestige de la sagesse déjà révélée du judaïsme et du christianisme.

\section{b) Fonction excluante}

Dans le même temps, la forme catalogique va permettre à certains auteurs de se livrer au jeu de l'exclusion. Ils s'emparent alors de la même forme catalogique pour opposer la prétendue sagesse des Grecs à une sagesse barbare plus ancienne et donc plus authentique.

Flavius Josèphe est l'un des premiers à opérer un renversement du rapport entre Grecs et Barbares, ce qui le mène à surdéterminer des traditions étrangères aux Grecs, désormais revendiquées comme supérieures à l'hellénisme ${ }^{35}$.

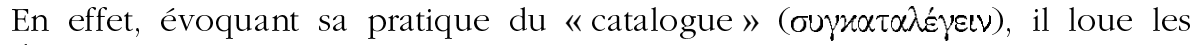
Égyptiens, les Chaldéens, les Phéniciens (et, implicitement son propre peuple) pour avoir préservé par écrit leurs traditions, contrairement aux Grecs ${ }^{36}$.

\footnotetext{
33 Aug., Ciu. VIII, 9 : Quicumque igitur philosophi de Deo summo et uero ista senserunt, quod et rerum creatarum sit effector et lumen cognoscendarum..., siue Platonici accommodatius nuncupetur, siue quodlibet aliud sectae suae nomen inponant; siue tantummodo Ionici generis, qui in eis praecipui fuerunt, ista senserint, sicut idem Plato ...; siue etiam Italicim propter Pythagoram...; siue aliarum quoque gentium qui sapientes uel philosophi habiti sunt, Atlantici Libyes, Aegypti, Indi, Persae, Chaldaei, Scythae, Galli, Hispani, aliqui reperiuntur qui boc uiderint ac docuerint: eos omnes ceteris anteponimus eosque nobis propinquiores fatemur.

${ }^{34}$ Arnobe, Adversus nationes IV, 13.

${ }^{35}$ Sur ce renversement, voir G.G. STROUMSA, Barbarian Philosophy: the Religious Revolution of Early Christianity, Tübingen, Mohr Siebeck, 1999.

${ }^{36}$ Jos., C. Ap. I, 8 (avec d'évidentes allusions au Timée et au Phèdre).
} 
Poursuivant le projet de Josèphe, les apologistes chrétiens vont intensément exploiter le thème de supériorité barbare, et en particulier hébraïque, et notamment l'élargir au domaine de la théologie et de l'éthique. Si les Grecs ont puisé en tout chez les Barbares pour ce qui est des sciences et des inventions pratiques, c'est surtout chez les Hébreux qu'ils ont puisé leur sagesse et une conception de Dieu conforme à la vérite $e^{-37}$.

L'idée de la dépendance, voire du larcin, des Grecs a la part belle dans les débats interculturels et interreligieux qui opposent les juifs, puis les chrétiens, aux paiens ${ }^{38}$. Pour Tatien, Clément et Eusèbe, les Grecs ont emprunté, et même volé des doctrines et des inventions pratiques aux Barbares, dont ils égrènent les noms sous forme d'un catalogue ${ }^{39}$. Cette idée découle directement de l'antiquité, et par conséquent, l'excellence de peuples non grecs ${ }^{40}$.

Dans un développement au sein duquel il revoit la chronologie du monde habité, Clément d'Alexandrie cite une foule de peuples qu'il considère comme sages et ce afin de montrer leur antériorité par rapport à la philosophie grecque et la dépendance de celle-ci à leur égard ${ }^{41}$ : Égyptiens, Babyloniens, Indiens, Mages, Brahmanes, Odryses, Gètes, Chaldéens, Arabes, Palestiniens, Hyperboréens et «mille nations encore », sont mentionnés dans ce contexte. Cependant, s'empresse-t-il d'ajouter, «le plus antique de beaucoup de tous ces peuples est le peuple juif et le fait que leur philosophie écrite est antérieure à la philosophie grecque est démontré avec maintes preuves par Philon [...] et Aristobule [...]» ${ }^{42}$. Les Grecs, eux, sont non seulement exclus, mais aussi placés en opposition aux termes de ce catalogue.

À la même époque environ, Celse propose lui aussi un catalogue de peuples sages les plus anciens dans des termes proches de celui de Clément, mais qui omet, cette fois, les juifs ${ }^{43}$. D'après l'auteur, y sont rassemblés ceux

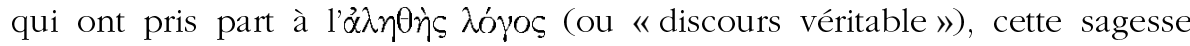
primitive que les platoniciens essayaient de découvrir par le biais de Platon et de certaines sagesses orientales. Il n'est pas à douter que Celse, dont on connait les attaques contre le judaïsme et le christianisme, ait intentionnellement écarté Moïse et les juifs de son catalogue.

Le catalogue de Celse comprend en outre des peuples inhabituellement cités, comme les Hyperboréens et les Odryses ainsi que les Éleusiniens et les habitants de Samothrace. Leur présence est intéressante dans la mesure où elle suggère que Celse désirait ouvrir le catalogue des peuples sages à des

\footnotetext{
${ }^{37}$ Par exemple, Eus., Prép. év. X, 4, 2-33.

${ }^{38}$ Voir D. Ridings, The Attic Moses. The Dependency Theme in Some Early Christian Writers, Göteborg, Acta Universitatis, 1995 (Studia graeca et latina gothoburgensia, 59).

${ }^{39}$ Tatien, Or. ad Gr. I, 1-3; Clém., Str. VI, 4, 3-5, 2; 16, 1; 27, 5-22; Eus., Prép. év. X, 4.

${ }^{40}$ Voir A.J. DROGE, Homer or Moses? Early Christian Interpretations of the History of Culture, Tübingen, Mohr Siebeck, 1989 (Hermeneutische Untersuchungen zur Theologie, 26).

${ }^{41}$ Clém., Str. I, 15, 68, 3 - 72, 4 .

${ }^{42}$ Clém., Str. I, 15, 72, 4 .

${ }^{43}$ Orig., C. C. I, 14.
} 
traditions plus grecques. Alors que le catalogue est traditionnellement constitué de nations, Celse le régionalise afin de l'élargir à certaines cités grecques célèbres pour leurs mystères : Éleusis et Samothrace.

La stratégie d'exclusion de Celse ne passe pas inaperçue dans le monde chrétien : Origène lui reproche d'avoir omis les juifs de son catalogue afin de délégitimiser la sagesse juive et celle des chrétiens ${ }^{44}$. En insistant sur la continuité entre judaïsme et christianisme, il s'agissait de montrer que ce dernier n'était pas une innovation dépourvue de crédit philosophique en raison de son caractère récent et novateur ${ }^{45}$.

Origène, contre-attaquant, s'étonne de ne pas trouver mention des juifs aux côtés des Égyptiens, Indiens et Assyriens. Il se dit également surpris de voir Celse mentionner ces peuples inhabituels dont il rejette l'inclusion intempestive. Cette manière de présenter les choses est révélatrice dans la mesure où le théologien classe lui-même les juifs parmi ces peuples orientaux traditionnellement associés.

Le catalogue des peuples sages sert donc à revendiquer la supériorité des sagesses barbares en général, et plus particulièrement à valoriser la sagesse hébraïque comme ultime source de vérité théologique.

Cependant, la fonction excluante à l'égard des Grecs n'a pas été exploitée exclusivement par des apologistes juifs et chrétiens. Ainsi, le néoplatonicien Porphyre a recueilli dans sa Philosophie tirée des oracles cette fameuse prophétie :

escarpée, très ardue est la route des bienheureux,

en premier lieu, elle est ouverte par des piliers de bronze,

il s'y trouve des sentiers inimaginables,

qu'ont fait voir pour l'accomplissement infini d'un oracle

les premiers mortels qui buvaient la belle eau du pays du Nil :

il y a aussi beaucoup de routes des bienheureux qu'ont connues les Phéniciens,

les Assyriens, les Lydiens et la race des hommes Hébreux (trad. É. des Places).

Ce document, qui constitue selon toute vraisemblance un authentique oracle d'Apollon $^{47}$, propose un catalogue de peuples qui auraient découvert la route menant au divin.

Contre toute attente, Porphyre, dont on connaît l'hostilité à l'égard des chrétiens, approuve l'enseignement de la prophétie et ne profite pas de la forme catalogique pour évincer les Hébreux des peuples sages. Dans son

\footnotetext{
${ }^{44}$ Orig., C. C. I, 15-16.

${ }^{45}$ Voir L.H. Feldman, «Origen's Contra Celsum and Josephus' Contra Apionem: The Issue of Jewish Origins », VChris 44 (1990), p. 105-135 et p. 196-197.

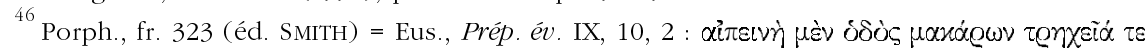

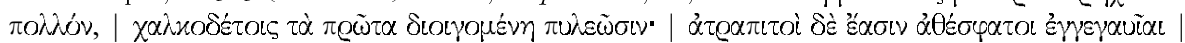

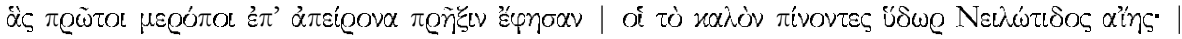

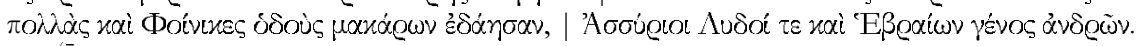

${ }^{47}$ Voir A. Busine, Paroles d'Apollon. Pratiques et traditions oraculaires dans l'antiquité tardive (II'-VI siècles), Leyde / Boston, Brill, 2005 (Religions in the Graeco-Roman World, 156), p. $214-216$
} 
commentaire à l'oracle, il rejette même la méthode grecque pour accéder au divin $^{48}$. À l'instar de Numénius et de Celse, Porphyre se tourne vers les peuples barbares pour recouvrer le véritable et antique Logos. Alors qu'il refuse cette voie aux Grecs de son époque, dans le De abstinentia, il n'en situe pas moins, sur un même plan, la race d'or des Grecs, les brahmanes et les mages au niveau de la piété, des mours et de la législation ${ }^{49}$.

Le procédé d'exclusion franchit ici une étape supplémentaire : les Grecs, sous-déterminés par rapport aux Barbares, ne sont pas simplement exclus du catalogue, mais explicitement dénigrés. Paradoxalement, cette position n'est pas éloignée de celle de chrétiens comme Clément ou Origène. À ceci près qu'aux yeux de Porphyre, les peuples ayant accès à la sagesse sont multiples, alors que ses adversaires les limitent à leurs ancêtres. Si la sagesse grecque, et en particulier le platonisme, constitue une première étape dans la quête de Dieu, c'est bien la tradition hébraïco-judéo-chrétienne qui est l'ultime source de sagesse ${ }^{50}$.

À l'encontre de la démarche de Porphyre, un autre champion de l'hellénisme, Diogène Laërce, que l'on situe traditionnellement aussi au milieu du III $^{\mathrm{e}}$ siècle, se réfère à un catalogue de peuples sages dans le but de réfuter la prétention de sagesse de peuples étrangers à la Grèce. En effet, Diogène s'oppose à ceux qui donnent à la philosophie une origine barbare, qu'elle soit égyptienne, perse, indienne, phénicienne, thrace ou encore libyenne ${ }^{51}$. Pour lui, la sagesse et la philosophie sont des phénomènes spécifiquement grecs, inventés par Musée à Athènes et par Linos à Thèbes, que mettront en pratique les Sept Sages puis les différentes écoles philosophiques grecques ${ }^{52}$. Dans ce cas, c'est le catalogue entier qui fait l'objet du processus d'exclusion.

Il est frappant de constater à quel point deux auteurs issus de la même sphère culturelle peuvent s'avérer opposés sur la question de la sagesse, mais aussi plus particulièrement dans leur utilisation des catalogues de peuples sages.

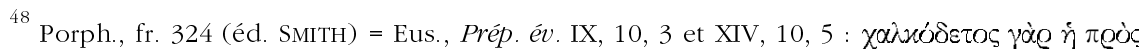

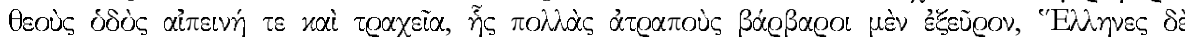

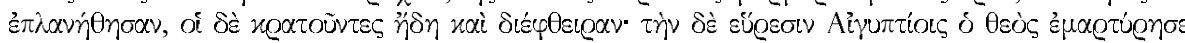

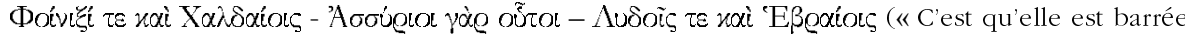
de bronze la route qui mène aux dieux, escarpée et ardue; les barbares en ont découvert bien des sentiers, mais les Grecs se sont égarés; ceux qui la tenaient à peine l'ont perdue; mais le dieu rend aux Égyptiens, aux Phéniciens, aux Chaldéens (ce sont les Assyriens), aux Lydiens et aux Hébreux, le témoignage qu'ils l'ont trouvée »). Voir Busine, o.c. (n. 47), p. 239, 266-268, 284.

${ }^{49}$ Voir Porph., De abst. IV.

${ }^{50}$ Sur ce thème, notamment chez Eusèbe, voir S. INOwLOCKI, Eusebius and the Jewish Authors. His Citation Technique in an Apologetic Context, Leyde / Boston, Brill, 2006 (Ancient Judaism and Early Christianity, 64), p. 105-138.

${ }^{51}$ DL. I, 1-2, 5-11.

52 DL. I, 3-4. Voir Busine, o.c. (n. 9), p. 53-56.
} 


\section{Conclusion}

Cette étude a établi les mécanismes de constitution des catalogues de peuples sages dans le monde gréco-romain et s'est concentrée sur leurs divers contextes d'exploitation. Nés dans le contexte politique et culturel propre aux conquêtes d'Alexandre, les catalogues ont par la suite été intégrés dans les démarches philosophiques et apologétiques des hellènes, des juifs et des chrétiens.

Plus fondamentalement, cet article éclaire, de façon inédite, les fonctions de ces catalogues dans les représentations de la transmission des savoirs, ainsi que dans les débats interreligieux et interculturels des premiers siècles de notre ère.

Il s'est avéré que, par leurs dimensions topologiques, chronologiques et généalogiques, les catalogues de peuples sages servent l'idée d'une sagesse morcelée dont il convient de recouvrer l'unité en recourant aux sagesses de peuples réputés.

Par ailleurs, en contexte apologétique et polémique, la mise en catalogue des peuples sages induit des fonctions d'inclusion et d'exclusion aussi subtiles qu'efficaces. Nous avons ainsi mis en évidence le lien intime entre le rôle de ces catalogues et la recherche de la sagesse et de la vérité divine chez les stoïciens et les platoniciens, et puis leur réappropriation par les chrétiens. Ces derniers substituent à l'idée d'une sagesse divine morcelée reconstituée par Platon celle des révélations juives et chrétiennes.

La mise en catalogue des peuples barbares fameux pour leur sagesse apparaît donc comme un phénomène important, non seulement pour comprendre la fonction épistémologique du catalogue, mais également pour approcher les problèmes identitaires et culturels de l'antiquité.

Michèle BROZE

Aude Busine

FNRS - Université libre de Bruxelles

Sabrina INOwLOCKI

50, av. Franklin Roosevelt

B - 1000 BRUXELLES

Courriels : sinowloc@ulb.ac.be

abusine@ulb.ac.be

mbroze@ulb.ac.be 